\title{
The Dynamic Queue Protocol for Spread Spectrum Random Access Networks
}

\author{
Qing Zhao and Lang Tong \\ School of Electrical and Computer Engineering \\ Cornell University, Ithaca, NY 14853 \\ \{qzhao,Itong\}@ee.cornell.edu
}

\begin{abstract}
The dynamic queue MAC protocol [12] is designed explicitly for networks with multipacket reception (MPR). It provides efficient channel utilization with a simple on-line implementation. In this paper, we present an analysis on the steady-state performance of the dynamic queue protocol. Its throughput and normalized throughput in CDMA networks are studied and compared to that of the optimal MAC [11] and the slotted ALOHA with optimal retransmission probability.
\end{abstract}

\section{INTRODUCTION}

One of the salient features of spread spectrum random access network is the enhanced user separation at the physical layer. Simultaneous transmissions from users with different signatures can be received reliably by the use of multiuser detection, space-time processing, or even simple matched filtering. In other words, spread spectrum networks support multipacket reception (MPR). Traditionally, MAC protocols are designed based on a collision channel model where any concurrent transmissions result in the destruction of all transmitted packets. The MPR capability of spread spectrum networks raises several important questions: (1) how does the MPR capability at the physical layer affect the performance of existing MAC protocols? (2) how should we design the MAC layer to fully exploit the MPR capability at the physical layer? Many researchers $[1,8,3,4,2]$ have addressed the first question. Answers to the second question, however, are scarce in the literature.

The Multi-Queue Service Room (MQSR) protocol proposed in [11] is perhaps the first MAC protocol designed explicitly for networks with MPR capability. It is optimal in terms of maximizing per-slot throughput and is capable of handling users with different QoS requirements. The drawback of the MQSR protocol, however, lies in its computational complexity. In [12], a dynamic queue protocol is proposed which achieves a performance comparable to

\footnotetext{
${ }^{0}$ This work was supported in part by the National Science Foundation under Contract CCR-9804019 and the Multidisciplinary University Research Initiative (MURI) under the Office of Naval Research Contract N00014-00-1-0564.
}

that of the MQSR protocol with an on-line implementation as simple as that of slotted ALOHA.

In this paper, we analyze the steady-state performance of the dynamic queue protocol. Its throughput and normalized throughput in CDMA networks are studied and compared to that of the MQSR protocol and the slotted ALOHA with optimal retransmission probability.

\section{THE MODEL}

We consider a communication network with $M$ users who transmit equal-sized packets to a central controller through a common wireless channel. Transmission time is slotted, and each packet requires one time slot to transmit. With probability $p$, a user independently generates a packet within each slot. The slotted channel is characterized by $C_{n, k}$, the probability of having $k$ successes in a slot with $n$ transmissions. The MPR matrix of the channel is given by

$$
\mathbf{C}=\left(\begin{array}{ccccc}
C_{1,0} & C_{1,1} & & & \\
C_{2,0} & C_{2,1} & C_{2,2} & & \\
\vdots & \vdots & \vdots & & \\
C_{M, 0} & C_{M, 1} & C_{M, 2} & \cdots & C_{M, M}
\end{array}\right)
$$

Let $\mathcal{C}_{n} \triangleq \sum_{k=1}^{n} k C_{n, k}$ be the expected number of correctly received packets when total $n$ packets are transmitted. We then define the capacity of an MPR channel as

$$
\eta \triangleq \max _{n=1, \cdots, M} \mathcal{C}_{n}
$$

Let $n_{0} \triangleq \arg \max _{n=1, \cdots, M} \mathcal{C}_{n}$. We can see that at heavy traffic load, $n_{0}$ packets should be transmitted simultaneously to achieve the channel capacity $\eta$. For MPR channels with $n_{0}$ greater than 1 , contention should be preferred at any traffic load in order to fully exploit the channel MPR capability.

We assume that the central controller can distinguish between empty and nonempty slots. If at least one packet is successfully demodulated at the end of a slot, the central controller does not assume the knowledge whether there are other packets transmitted in this slot but not successfully received. 


\section{THE DYNAMIC QUEUE PROTOCOL}

In the dynamic queue protocol, the time axis is divided into transmission periods (TPs). Each TP is dedicated to the transmission of packets generated in the previous TP and ends when the central controller can assert that all packets generated in the previous TP have been successfully transmitted. We assume that besides the packet waiting for transmission in the current TP, each user can hold at most one packet newly generated in the current TP and to be transmitted in the next one. Let $q_{i}$ denote the probability that a user has a packet to transmit in the $i$ th $(i \geq 1)$ TP. We have

$$
q_{i}=1-(1-p)^{L_{i-1}},
$$

where $L_{i-1}(i \geq 2)$ denotes the length of the $(i-1)$ th TP defined as the number of slots it contains; $L_{0}$ specifies the network initial condition and is known to the central controller.

At the beginning of the $i$ th TP, all $M$ users are waiting in a queue for the transmission of their packets generated in the $(i-1)$ th TP. Based on $q_{i}$ given by (3), $N_{i}$, the size of the access set for this TP, is chosen. Then, the first $N_{i}$ users in the queue are enabled to access the channel in the first slot of the $i$ th TP. At the end of this slot, the central controller detects whether this slot is empty or not. If it is empty, all these $N_{i}$ users are processed and the next $N_{i}$ users in the queue are enabled in the next slot. On the other hand, if this slot is not empty and $k(k \geq 0)$ packets are successfully received, the sources of these $k$ packets are processed and removed from the waiting queue; the rest $N_{i}-k$ users along with the next $k$ users in the queue are enabled to access the channel in the next slot. This procedure continues until all $M$ users are processed.

With this structure, the only parameter to be designed is $N_{i}$, which is chosen so that the expected length of the $i$ th TP is minimized, i.e.,

$$
N_{i}=\arg \min _{N=1, \cdots, M} E\left[L_{i} \mid N\right],
$$

where $E\left[L_{i} \mid N\right]$ is the expected length of the $i$ th TP when the size of the contention class is chosen to be $N$. It is shown in $[12,9]$ that $E\left[L_{i} \mid N\right]$ can be computed as the absorbing time of a finite state Markov chain. With $E\left[L_{i} \mid N\right]$ computed for all possible $N$, the optimal size $N_{i}$ of the access class for the $i$ th TP can be easily obtained from (4).

We point out that the optimal size of the access set can be computed off line and stored in a table. When the network starts, the optimal size of the access set for each TP can be obtained from this table; little on-line computation is required.

\section{STEADY-STATE PERFORMANCE ANALYSIS}

\subsection{The Existence of Steady State}

Given the channel reception matrix $\mathbf{C}$ and the incoming traffic load $p$, the optimal size $N_{i}$ of the access set for the $i$ th TP is a function of $L_{i-1}$, i.e., $N_{i}=f_{\mathbf{C}, p}\left(L_{i-1}\right)$. In general, $f_{\mathbf{C}, p}(\cdot)$ is a monotonic decreasing function as illustrated in Figure 1. It is completely determined by $\mathbf{C}$ and $p$ and can be computed off line. Suppose that the range of $f_{\mathbf{C}, p}(\cdot)$ is $\left\{\underline{n}_{1}, \cdots, \underline{n}_{J}\right\}$ with $\underline{n}_{1}<\underline{n}_{2}<\cdots<\underline{n}_{J}$. We then define

$$
\underline{l}_{j} \triangleq \min \left\{l: f_{\mathbf{C}, p}(l)=\underline{n}_{j}\right\}, \quad j=1, \cdots, J .
$$

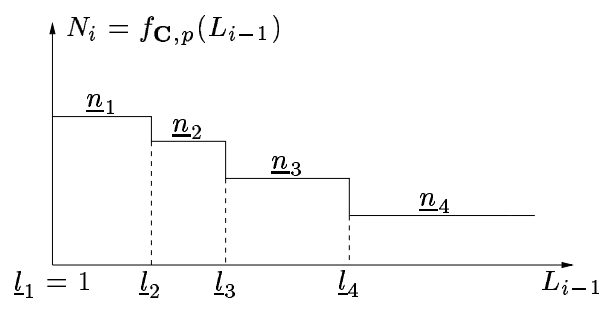

Figure 1: $N_{i}$ as a function of $L_{i-1}$.

It can be shown that $\left\{L_{i}\right\}_{i=0}^{\infty}$ is a homogeneous Markov process with positive integers as its infinite state space $\mathcal{S}$. The steady state of a network using the dynamic queue protocol is then defined as the stationary distribution of $\left\{L_{i}\right\}_{i=0}^{\infty}$. Before using steady-state performance measures such as throughput and average delay, questions about the existence and uniqueness of the network steady states must be resolved.

Theorem 1 Suppose that $f_{\mathbf{C}, p}(\cdot)$ is a monotone decreasing function with range $\left\{\underline{n}_{1}, \cdots, \underline{n}_{J}\right\}$. Let

$\mathcal{Q}_{1}=\left\{1,2, \cdots,\left\lceil\frac{M}{\underline{n}_{w}}\right\rceil-1\right\}, \quad \mathcal{Q}_{2}=\left\{\left\lceil\frac{M}{\underline{n}_{w}}\right\rceil,\left\lceil\frac{M}{\underline{n}_{w}}\right\rceil+1, \cdots\right\}$

be a partition of the state space $\mathcal{S}$, where

$$
w \triangleq \max \left\{j:\left\lceil\frac{M}{\underline{n}_{j}}\right\rceil \geq \underline{l}_{j}, 1 \leq j \leq J\right\} .
$$

Consider a noisy environment with $0<C_{1,0}<1$. We have, for $p \in(0,1)$,

T1.1 all states in $\mathcal{Q}_{1}$ are transient;

T1.2 if the initial distribution of $\left\{L_{i}\right\}_{i=0}^{\infty}$ is such that $P\left[L_{0} \in\right.$ $\left.\mathcal{Q}_{2}\right]=1$, then $\left\{L_{i}\right\}_{i=0}^{\infty}$ is ergodic;

T1.3 $\left\{L_{i}\right\}_{i=0}^{\infty}$ has a limiting distribution $\left\{\pi_{l}\right\}_{l \in \mathcal{S}}$ satisfying

$$
\pi_{l}\left\{\begin{array}{ll}
>0 & \text { if } l \in \mathcal{Q}_{2} \\
=0 & \text { if } l \in \mathcal{Q}_{1}
\end{array} .\right.
$$


The proof of Theorem 1 can be found in [9]. Theorem 1 shows that a network which employs the dynamic queue protocol will eventually reach a unique steady state, regardless of the initial condition $L_{0}$. Thus, we can use measures such as throughput and average delay to study the long term behavior of the dynamic queue protocol.

\subsection{Throughput and Packet Delay}

The throughput $U$ is defined as the average number of packets successfully transmitted within one time slot. Let $S_{i}$ denote the number of packets generated in the $i$ th TP. Recall that packets generated in the $i$ th TP are all successfully transmitted in the $(i+1)$ th TP. We have

$$
U=\lim _{i \rightarrow \infty} \frac{S_{0}+S_{1}+\cdots+S_{i-1}}{L_{1}+L_{2}+\cdots+L_{i}} .
$$

By Theorem 1, $\left\{L_{i}\right\}_{i=0}^{\infty}$ is an ergodic process with limiting distribution $\left\{\pi_{l}\right\}_{l \in \mathcal{S}}$ given by (8). Hence, at steady state, we have, for any $p \in(0,1)$,

$$
U=\frac{E\left[S_{i}\right]}{E\left[L_{i}\right]}=\frac{\sum_{l \in \mathcal{Q}_{2}}\left(1-(1-p)^{l}\right) M \pi_{l}}{\sum_{l \in \mathcal{Q}_{2}} l \pi_{l}} .
$$

The average packet delay $D$ is defined as the average number of slots from the time a packet is generated to that it is successfully transmitted. Since a packet generated in the $i$ th TP is transmitted in the $(i+1)$ th TP, the average packet delay is determined by the lengths of two consecutive TPs. Based on the Markovian property of $\left\{L_{i}\right\}_{i=0}^{\infty}$, we can show that $\left\{\left(L_{i}, L_{i+1}\right)\right\}_{i=0}^{\infty}$ is also a homogeneous Markov process with state space $\hat{\mathcal{S}} \in \mathcal{S} \times \mathcal{S}$. Furthermore, if $\left\{L_{i}\right\}_{i=0}^{\infty}$ has a limiting distribution $\left\{\pi_{l}\right\}_{l \in \mathcal{S}}$ given by (8), then $\left\{\left(L_{i}, L_{i+1}\right)\right\}_{i=0}^{\infty}$ has a limiting distribution

$$
\hat{\pi}_{(l, m)}=\pi_{l} p_{l, m}\left\{\begin{array}{ll}
>0 & \text { if } l, m \in \mathcal{Q}_{2} \\
=0 & \text { otherwise }
\end{array},\right.
$$

where $p_{l, m} \triangleq P\left[L_{i}=m \mid L_{i-1}=l\right]$ is the transition probability of $\left\{L_{i}\right\}_{i=0}^{\infty}$.

In the steady state, with probability $\hat{\pi}_{(l, m)}$, a packet is generated in a TP with length $l$ and successfully transmitted in a TP with length $m$. Without loss of generality, we assume these two transmission periods are the first and the second TP. Let $t_{g}$ and $t_{s}$ denote, respectively, the time instance that the packet is generated and that the packet is successfully received. Assuming that the first TP starts at time 0 and each slot lasts one time unit, we have

$$
\begin{aligned}
D & =\sum_{l, m \in \mathcal{Q}_{2}} E\left[t_{s}-t_{g} \mid L_{1}=l, L_{2}=m\right] \hat{\pi}_{(l, m)} \\
& =\sum_{l, m \in \mathcal{Q}_{2}}\left(E\left[t_{s} \mid L_{1}=l, L_{2}=m\right]-E\left[t_{g} \mid L_{1}=l\right]\right) \hat{\pi}_{(l, m)} \\
& \leq \sum_{l, m \in \mathcal{Q}_{2}}\left(l+m-\frac{p \sum_{k=1}^{l} k(1-p)^{k-1}}{1-(1-p)^{l}}+0.5\right) \pi_{l} p_{l, m},(12)
\end{aligned}
$$

where we have assumed that $t_{g}$ is uniformly distributed in the slot in which the packet is generated.
From $(10,12)$ we see that the throughput and average delay provided by the dynamic queue protocol are given by the limiting distribution $\left\{\pi_{l}\right\}_{l \in \mathcal{S}}$ and the transition probability $\left\{p_{l, m}\right\}_{l, m \in \mathcal{S}}$ of $\left\{L_{i}\right\}_{i=0}^{\infty}$. In general, these two quantities are difficult to obtain even numerically. For simple examples, however, they may be studied analytically as shown in Section 5.1.

\section{NUMERICAL AND SIMULATION EXAMPLES}

\subsection{A Numerical Example}

We first study a simple numerical example with $M=2$. The channel reception matrix is given by

$$
\mathbf{C}=\left(\begin{array}{lll}
1-p_{1} & p_{1} & 0 \\
1-p_{2} & p_{2} & 0
\end{array}\right)
$$

where $0<p_{1}, p_{2} \leq 1$. By analyzing the absorbing time of a finite-sate Markov chain, we have

$$
\begin{aligned}
& E\left[L_{i} \mid q_{i}, N_{i}=1\right]=2+2\left(1-p_{1}\right) q_{i} / p_{1}, \\
& E\left[L_{i} \mid q_{i}, N_{i}=2\right]=1+\frac{2}{p_{1}} q_{i}+\frac{p_{1}-p_{2}-p_{1} p_{2}}{p_{1} p_{2}} q_{i}^{2} .
\end{aligned}
$$

Thus, to minimize $E\left[L_{i}\right]$, we have, for the case of $p_{2}<p_{1}$,

$$
N_{i}=\left\{\begin{array}{ll}
2 & \text { if } q_{i} \leq q^{*} \\
1 & \text { if } q_{i}>q^{*}
\end{array},\right.
$$

where

$$
q^{*}=\left\{\begin{array}{ll}
\frac{1}{2} & \text { if } p_{2}=\frac{p_{1}}{1+p_{1}} \\
\frac{\sqrt{p_{1} p_{2}\left(p_{1}-p_{2}\right)}-p_{1} p_{2}}{p_{1}-p_{2}-p_{1} p_{2}} & \text { otherwise }
\end{array} .\right.
$$

By taking into account the incoming traffic load $0<p<1$, $N_{i}$ as a function of $L_{i-1}$ can be obtained from (15) as

$$
N_{i}= \begin{cases}2 & \text { if } 1 \leq L_{i-1}<\underline{l}_{2} \\ 1 & \text { if } L_{i-1} \geq \underline{l}_{2}\end{cases}
$$

where

$$
\underline{l}_{2}=\left\lceil\frac{\ln \left(1-q^{*}\right)}{\ln (1-p)}\right\rceil .
$$

Suppose that $p \geq 1-\sqrt{1-q^{*}}$. In this case, we have $\underline{l}_{2} \leq 2$. From Theorem 1 we conclude that

$$
\mathcal{Q}_{1}=\{1\}, \quad \mathcal{Q}_{2}=\{2,3, \cdots\}
$$

contain, respectively, the transient states and positive recurrent states of $\left\{L_{i}\right\}_{i=0}^{\infty}$. It can be shown that the transition probability for states in $\mathcal{Q}_{2}$ is given by

$$
p_{l, m}=\left\{\begin{array}{l}
\left(1-(1-p)^{l}\right)^{2} p_{1}^{2}+2(1-p)^{l}\left(1-(1-p)^{l}\right) p_{1} \\
+(1-p)^{2 l}(\text { if } m=2) \\
\left(1-(1-p)^{l}\right)^{2}(m-1) p_{1}^{2}\left(1-p_{1}\right)^{m-2} \\
+2(1-p)^{l}\left(1-(1-p)^{l}\right) p_{1}\left(1-p_{1}\right)^{m-2} \\
(\text { if } m>2)
\end{array}\right.
$$

The limiting distribution of $\left\{L_{i}\right\}_{i=0}^{\infty}$ is then given by

$$
\pi_{l}=\left\{\begin{array}{ll}
a p_{1}^{2}+b p_{1}+(1-a-b) & \text { if } l=2 \\
a(l-1) p_{1}^{2}\left(1-p_{1}\right)^{l-2}+b p_{1}\left(1-p_{1}\right)^{l-2} & \text { if } l>2
\end{array},\right.
$$


where $a$ and $b$ are, respectively, the probability that both users have packet and that only one user has packet at the beginning of a TP in the steady state. It is difficult to obtain them in close form. However, by estimating them through simulations, we can easily obtain the limiting distribution of $\left\{L_{i}\right\}_{i=0}^{\infty}$. The throughput can then be calculated from (10).

We now consider an example with $p_{1}=\frac{3}{4}$ and $p_{2}=\frac{1}{2}$. The limiting distribution of $\left\{L_{i}\right\}_{i=0}^{\infty}$ at $p=0.4$ and $p=0.9$ are shown in Figure 2-left, where we see that $\pi_{l}$ decays exponentially in $l$, as promised by (21). Comparing the limiting distribution at $p=0.4$ and that at $p=0.9$, we see that $E\left[L_{i}\right]$ increases with the incoming traffic load $p$. The calculated and the simulated throughput of this 2-user system are shown in Figure 2-right as a function of $p$. In both figures, the theoretical results match perfectly with the simulation results.
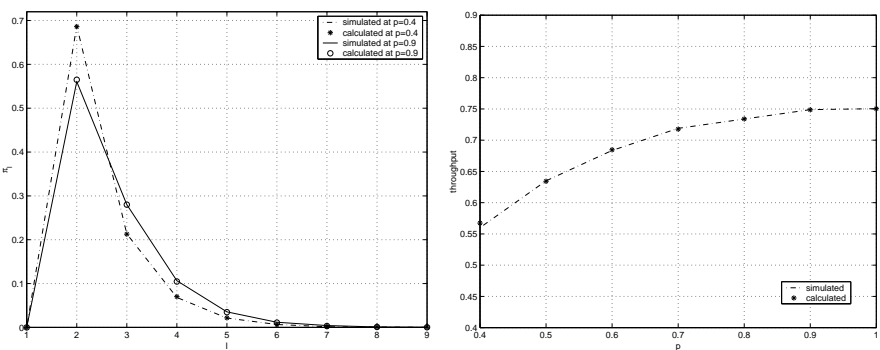

Figure 2: The 2-user example.

\subsection{Simulation Examples: MPR via Spread Spec- trum}

In this example, we consider a CDMA network with $M$ users. Each transmitted packet is spread by a randomly generated code with length $P$. At the central controller, the spreading code of each transmitted packet is assumed known, and a bank of matched filters are used as the receiver. We assume that each packet contains $L_{p}$ bits. A block error control code is used which corrects up to $t$ errors in each received packet. We consider a noisy environment where the variance of the additive white Gaussian noise is denoted by $\sigma^{2}$.

We first construct the reception matrix $\mathbf{C}$ for such a network. Under the Gaussian assumption on the multiaccess interference from users with equal power, the BER $p_{e}$ of a packet received in the presence of $n-1$ interfering packets is given by [6]

$$
p_{e}(n-1)=Q\left(\sqrt{\frac{3 P}{n-1+3 P \sigma^{2}}}\right) .
$$

Assuming that errors occur independently in a packet, we then have the packet success probability in the presence of $n-1$ interfering packets as

$$
p_{s}(n-1)=\sum_{i=0}^{t} B\left(i, L_{p}, p_{e}\right) .
$$

Under the assumption that each matched filter works independently at the receiver, we have

$$
C_{n, k}=B\left(k, n, p_{s}(n-1)\right)
$$

Throughput In this example, we compare the throughput performance of the dynamic queue protocol with that of the MQSR protocol and the slotted ALOHA with optimal retransmission probability. We considered a network with $M=10$. The packet length $L_{p}$, spreading gain $P$, and the number of correctable errors in a packet were, respectively, 200,6 , and 2 . The noise variance was given by $10 \log _{10} \frac{1}{\sigma^{2}}=10 d B$. The capacity of the MPR channel in such a network is 1.7925 , which can be achieved by transmitting $n_{0}=2$ packets in each slot.

We first construct the look-up table that specifies the $q_{i}$ intervals in which a possible size (from 1 to 10) of access set is optimal. The result is shown in Figure 3-left. This result demonstrates clearly the trend that the heavier the traffic is (larger $q_{i}$ ), the smaller the access set should be, as intuition suggests. Note that the optimal size of access set equals to $n_{0}$ which is greater than 1 at the heaviest traffic load $\left(q_{i}=1\right)$, indicating that contention is preferable at any traffic load for this MPR channel.
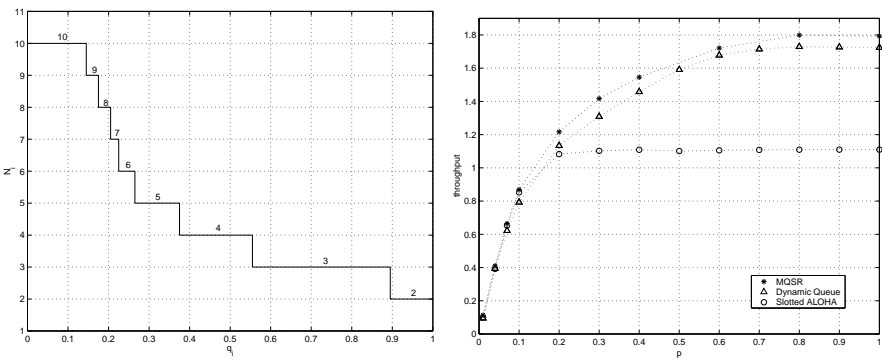

Figure 3: Throughput comparison.

In Figure 3-right, the throughput performance of the dynamic queue protocol at different incoming traffic load $p$ is compared to that of the MQSR protocol [11] and the slotted ALOHA with delayed first transmission. Here we intentionally favored the slotted ALOHA by letting it choose the optimal retransmission probability. Comparing the performance of the dynamic queue protocol with that of the slotted ALOHA with optimal retransmission probability, we see a $55 \%$ throughput gain at medium and heavy traffic load. Compared to the optimal MQSR protocol, the dynamic queue protocol achieved comparable performance with a much simpler implementation. Note that the throughput provided by the dynamic queue protocol at heavy traffic load approached to the channel capacity.

Normalized Throughput While the techniques of spread spectrum and error control strengthen the channel reception capability, they consume bandwidth. In this example, we study the normalized throughput of the dynamic queue 
protocol, where we define the normalized throughput as the average number of information bits successfully transmitted per second per Hertz [7]. We assume here a BPSK modulation. Given the network throughput $U$, spreading gain $P$, packet length $L_{p}$, coding rate $r_{c}$, and symbol duration $T_{s}$, the average number of successfully transmitted information bits per slot is $L_{p} r_{c} U$; the duration of each time slot is $L_{p} T_{s}$ and the bandwidth $\frac{P}{T_{s}}$. Hence, the normalized throughput $U_{n}$ is given by

$$
U_{n}=\frac{L_{p} r_{c} U}{L_{p} T_{s} \frac{P}{T_{s}}}=\frac{r_{c}}{P} U
$$

As shown in [5], the maximum coding rate $r_{c}$ can be computed from the number $t$ of correctable errors as follows.

$$
\begin{aligned}
\alpha & =\frac{2 t+1}{L_{p}} \\
r_{c} & =1+\alpha \log _{2}(\alpha)+(1-\alpha) \log _{2}(1-\alpha) .
\end{aligned}
$$

We compare the normalized throughput of the dynamic queue protocol with that of the MQSR protocol and the slotted ALOHA with optimal retransmission probability. We choose $p=1$ for the reason that all three protocols yield maximum throughput at this heaviest traffic load. The network parameters were chosen as $M=200, L_{p}=$ $1000, P=10$, and $\sigma^{2}=0$. The normalized throughput of the dynamic queue, the MQSR, and the slotted ALOHA with optimal retransmission probability at $p=1$ was theoretically calculated and plotted in Figure 4 as $t$, the number of correctable errors within one packet, varies from 0 to 150 . From Figure 4, we again observe that the dynamic queue protocol performed comparably to the optimal MQSR protocol and significantly better than the slotted ALOHA with optimal retransmission probability. Note that the MQSR protocol achieves the channel capacity at $p=1$ which has been shown theoretically in [10]. A comparable performance to it implies that the throughput provided by the dynamic queue protocol approaches to the channel capacity at heavy traffic load. Furthermore, Figure 4 shows that to achieve the best bandwidth efficiency, we should choose a block error control code which corrects up to $t=30$ errors out of a packet with 1000 bits for the dynamic queue and the MQSR protocol. For the slotted ALOHA with optimal retransmission probability, however, we should choose $t=60$. A block error control code with stronger correction capability is, in general, more difficult to design.

\section{CONCLUSION}

In this paper, an analysis on the steady-state performance of the dynamic queue protocol is presented. Simulation results demonstrate that the dynamic queue protocol is superior to the slotted ALOHA with optimal retransmission probability in the efficiency of utilizing the channel MPR

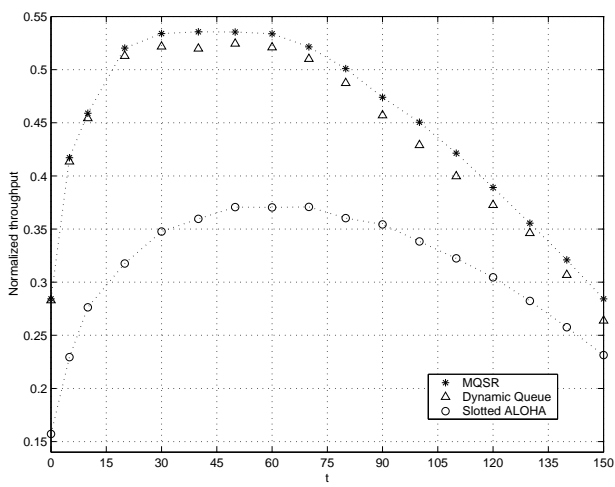

Figure 4: Normalized throughput at $p=1$.

capability. It achieves a performance comparable to that of the optimal MQSR protocol with a simple implementation.

\section{REFERENCES}

[1] N. Abramson. "The Throughput of Packet Broadcasting Channels". IEEE Trans. Comm, COM-25(1):117-128, January 1977.

[2] J.Q. Bao and L. Tong. "A Performance Comparison Between Ad Hoc and Centrally Controlled CDMA Wireless LANs". Submitted to IEEE J. Select. Areas Commun., 2000.

[3] S. Ghez, S. Verdú, and S.C. Schwartz. "Stability Properties of Slotted Aloha with Multipacket Reception Capability" . IEEE Trans. Automat. Contr., 33(7):640-649, July 1988.

[4] S. Ghez, S. Verdú, and S.C. Schwartz. "Optimal Decentralized Control in the Random Access Multipacket Channel". IEEE Trans. Automat. Contr., 34(11):1153-1163, Nov. 1989.

[5] R.K. Morrow Jr. and J.S. Lehnert. "Packet Throughput in Slotted ALOHA DS/SSMA Radio Systems with Random Signature Sequences". IEEE Trans. Comm., 40(7):1223-1230, July 1992.

[6] J. Lehnert and M. Pursley. “Error Probabilities for Binary Direct-Sequence Spread-Spectrum Communications with Random Signature Sequences". IEEE Trans. on Communications, COM-35(1):87-98, January 1987.

[7] A. Polydors and J. Sylvester. "Slotted Random Access SpreadSpectrum Networks: An Analytical Framework". IEEE Journal on Selected Areas in Communications, SAC-5:989-1002, July 1987.

[8] M. Sidi and I. Cidon. "Splitting Protocols in Presence of Capture". IEEE Trans. Info. Theory, IT-31:295-301, March 1985.

[9] Q. Zhao and L. Tong. "A Dynamic Queue Protocol for Multiaccess Wireless Networks with Multipacket Reception". Submitted to IEEE/ACM Transactions on Networking.

[10] Q. Zhao and L. Tong. "A Multi-Queue Service Room MAC Protocol for Wireless Networks with Multipacket Reception". Submitted to IEEE/ACM Transactions on Networking.

[11] Q. Zhao and L. Tong. "An Adaptive Multi-Queue Service Room Protocol for Wireless Networks with Multipacket Reception". In Proc. 2000 Allerton Conference on Communications, Control, and Computing, Urbana, IL, Oct. 2000.

[12] Q. Zhao and L. Tong. "The Dynamic Queue Protocol for Multiaccess Networks with Multipacket Reception". In Proc. 34th Asilomar Conf. on Signals, Systems, and Comp., Nov. 2000. 